\title{
Quantile Regression in Risk Calibration
}

\author{
Shih-Kang Chao* \\ Wolfgang Karl Härdle* \\ Weining Wang*
}

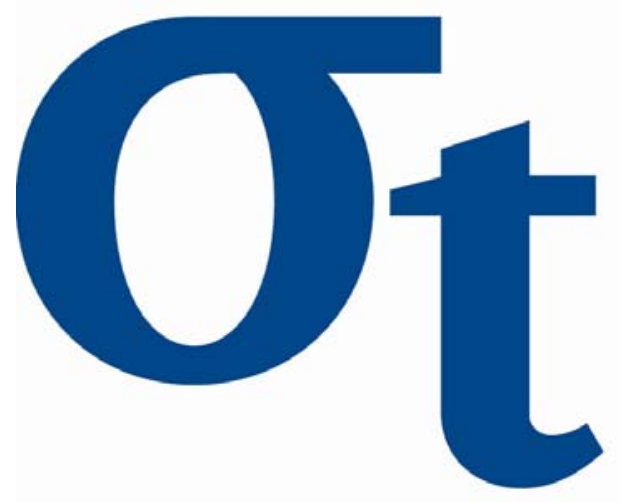

* Humboldt-Universität zu Berlin, Germany 


\title{
Quantile Regression in Risk Calibration*
}

\author{
Shih-Kang Chaoł Wolfgang Karl Härdleł Weining Wang§
}

January 24, 2012

\begin{abstract}
Financial risk control has always been challenging and becomes now an even harder problem as joint extreme events occur more frequently. For decision makers and government regulators, it is therefore important to obtain accurate information on the interdependency of risk factors. Given a stressful situation for one market participant, one likes to measure how this stress affects other factors. The CoVaR (Conditional VaR) framework has been developed for this purpose. The basic technical elements of CoVaR estimation are two levels of quantile regression: one on market risk factors; another on individual risk factor.

Tests on the functional form of the two-level quantile regression reject the linearity. A flexible semiparametric modeling framework for CoVaR is proposed. A partial linear model (PLM) is analyzed. In applying the technology to stock data covering the crisis period, the PLM outperforms in the crisis time, with the justification of the backtesting procedures. Moreover, using the data on global stock markets indices, the analysis on marginal contribution of risk (MCR) defined as the local first order derivative of the quantile curve sheds some light on the source of the global market risk.
\end{abstract}

Keywords: CoVaR, Value-at-Risk, quantile regression, locally linear quantile regression, partial linear model, semiparametric model

JEL classification: C14, C21, C22, C53, G01, G10, G20, G32

\section{Introduction}

Sufficiently accurate risk measures are needed not only in crisis times. In the last two decades, the world has gone through several financial turmoils, and the financial market is getting riskier and the scale of loss soars. Beside marginal extremes that can shock even a well diversified portfolio, the focus of intensified research in the recent years has been on understanding the interdependence of risk factors and their conditional structure.

*The financial support from the Deutsche Forschungsgemeinschaft via SFB 649 "Ökonomisches Risiko", HumboldtUniversität zu Berlin is gratefully acknowledged.

${ }^{\dagger}$ Research associate at Ladislaus von Bortkiewicz Chair, the Institute for Statistics and Econometrics of HumboldtUniversität zu Berlin, Spandauer Straße 1, 10178 Berlin, Germany. Email: shih-kang.chao@cms.hu-berlin.de

${ }^{\ddagger}$ Professor at Humboldt-Universität zu Berlin and Director of C.A.S.E. - Center for Applied Statistics and Economics, Humboldt-Universität zu Berlin, Spandauer Straße 1, 10178 Berlin, Germany. Email: haerdle@wiwi.huberlin.de

${ }^{\S}$ Research associate at Ladislaus von Bortkiewicz Chair, the Institute for Statistics and Econometrics of HumboldtUniversität zu Berlin, Spandauer Straße 1, 10178 Berlin, Germany. Email: wangwein@cms.hu-berlin.de 
The most popular risk measure is the Value-at-Risk (VaR), which is defined as the $\tau$-quantile of the return distribution at time $t+d$ conditioned on the information set $\mathcal{F}_{t}$ :

$$
V a R_{t+d}^{\tau} \stackrel{\text { def }}{=} \inf \left\{x \in \mathbb{R}: \mathrm{P}\left(X_{t+d} \leq x \mid \mathcal{F}_{t}\right) \geq \tau\right\}
$$

Here $X_{t}$ denotes the asset return and $\tau$ is taking values such as $0.05,0.01$ or 0.001 to reflect negative extreme risk.

Extracting information in economic variables to predict VaR brings quantile regression into play here, since VaR is the quantile of the conditional asset return distribution. Engle and Manganelli (2004) propose the nonlinear Conditional Autoregressive Value at Risk (CaViaR) model, which uses (lag) VaR and lag returns. Chernozhukov and Umantsev (2001) propose linear and quadratic time series models for VaR prediction. Kuan et al. (2009) propose the Conditional AutoRegressive Expectile (CARE) model, and argue that expectiles are more sensitive to the scale of losses. These studies and many others apply quantile regression in a prespecified often linear functional form. In a more nonparametric context, Cai and Wang (2008) estimate the conditioned cdf by a double kernel local linear estimator and find the quantile by inverting the cdf. Schaumburg (2011) uses the same technique together with extreme value theory for VaR prediction. Taylor (2008) proposes Exponentially Weighted Quantile Regression (EWQR) for estimating VaR time series.

The aforementioned studies focus mainly on the VaR estimation for single assets and do not directly take into account the escalated spillover effect in crisis periods. This risk of joint tail events of asset returns has been identified and studied. Further, Brunnermeier and Pedersen (2008) show that the negative feedback effect of a "loss spiral" and a "margin spiral" leads to the joint depreciation of assets prices. It is therefore important to develop risk measures which can quantify the contagion effects of negative extreme event.

Acharya et al. (2010) propose the concept of marginal expected shortfall (MES), which measures the contribution of individual assets to the portfolio expected shortfall. Via an equilibrium argument, the MES is shown to be a predictor to a financial institution's risk contribution. Brownlees and Engle (2010) demonstrate that the MES can be written as a function of volatility, correlation and expectation conditional on tail events. Huang et al. (2011) propose the distress insurance premium (DIP), a measure similar to MES but computed under the risk-neutral probability. This measure can therefore be viewed as the market insurance premium against the event that the portfolio loss exceeds a low level. Adams et al. (2010) construct financial indices on return of insurance companies, commercial banks, investment banks and hedge funds, and use a linear model for the VaRs of the four financial indices to forecast the state-dependent sensitivity VaR (SDSVaR). The risk measures proposed above have some shortcomings though: The computation of DIP is demanding since this involves the simulation of rare events. MES suffers from the scarcity of data because it conditions on a rare event.

In Adrian and Brunnermeier (2011) (henceforth AB), the CoVaR concept of conditional VaR is proposed, which controls the effect of the negative extreme event of some systemically risky financial institutions. Formally, let $C\left(X_{i, t}\right)$ be some event of a asset $i$ return $X_{i, t}$ at time $t$ and take $X_{j, t}$ as another asset return (e.g. the market index). The $\operatorname{CoVaR}_{j \mid i, t}^{\tau}$ is defined as the $\tau$-quantile of 
the conditional probability distribution:

$$
\mathrm{P}\left\{X_{j, t} \leq \mathrm{CoVaR}_{j \mid i, t}^{\tau} \mid C\left(X_{i, t}\right), M_{t}\right\}=\tau,
$$

where $M_{t}$ is a vector of market variables defined in Section 2.1. The standard CoVaR approach is to set $C\left(X_{i, t}\right)=\left\{X_{i, t}=V a R_{X_{i, t}}^{\tau}\right\}$. In $\mathrm{AB}, X_{j, t}$ is the weekly return which is constructed from a vast data set comprised of all publicly traded commercial banks, broker dealers, insurance companies, and real estate companies in the U.S. Further, $\mathrm{AB}$ propose $\triangle \mathrm{CoVaR}$ (measure of marginal risk contribution) as the difference between $C o V a R_{j \mid i, t}^{\tau_{1}}$ and $C o V a R_{j \mid i, t}^{\tau_{2}}$, where $\tau_{1}=0.5$ associated with the normal state and $\tau_{2}=0.05$ associated with the financial distress state.

The formulation of this conditional risk measure has several advantages. First, the cloning property: After dividing a systemically risky firm into several clones, the value of CoVaR conditioned on the entire firm does not differ from the one conditioned on one of the clones. Second, the conservativeness. The CoVaR value is more conservative than VaR because it conditions on an extreme event. Third, CoVaR is endogenously generated and adapted to the varying environment of the market.

The recipe of $\mathrm{AB}$ for $\mathrm{CoVaR}$ construction is as follows: In a first step one predicts the VaR of an individual asset $X_{i, t}$ through a linear model on market variables:

$$
X_{i, t}=\alpha_{i}+\gamma_{i}^{\top} M_{t-1}+\varepsilon_{i, t},
$$

where $\gamma_{i}^{\top}$ means the transpose of $\gamma_{i}$ and $M_{t}$ is a vector of the state variables (see Section 2.1). This model is estimated with quantile regression of Koenker and Bassett (1978) to get the coefficients $\left(\hat{\alpha}_{i}, \hat{\gamma}_{i}\right)$ with $F_{\varepsilon_{i, t}}^{-1}\left(\tau \mid M_{t-1}\right)=0$. The VaR of asset $i$ is predicted by

$$
\widehat{V a R}_{i, t}=\hat{\alpha}_{i}+\hat{\gamma}_{i}^{\top} M_{t-1} \text {. }
$$

In a second step one models the asset $j$ return as a linear function of asset return $i$ and market variables $M_{t}$ :

$$
X_{j, t}=\alpha_{j \mid i}+\beta_{j \mid i} X_{i, t}+\gamma_{j \mid i}^{\top} M_{t-1}+\varepsilon_{j, t}
$$

again one employs quantile regression and obtains coefficients $\left(\hat{\alpha}_{j \mid i}, \hat{\beta}_{j \mid i}, \hat{\gamma}_{j \mid i}\right)$. The CoVaR is finally calculated:

$$
\widehat{C o V a R}_{j \mid i, t}^{A B}=\hat{\alpha}_{j \mid i}+\hat{\beta}_{j \mid i} \widehat{\operatorname{VaR}}_{i, t}+\hat{\gamma}_{j \mid i}^{\top} M_{t-1}
$$

In equation (5) the variable $X_{i, t}$ influences the return $X_{j, t}$ in a linear fashion. However, the linear parametric model may not be flexible enough to capture the tail dependence between $i$ and $j$. The linearity of the conditioned quantile curves of $X_{j}$ on $X_{i}$ is challenged by the confidence bands of the nonparametric quantile curves, as shown in Figure 1.1. The left tail quantile from linear parametric quantile regression (red) lies well outside the confidence band (gray dashed curve) of Härdle and Song (2010). This motivates empirically, that a linear model is not flexible enough for the CoVaR question at hand.

Nonparametric models can be used to account for the nonlinear structure of the conditional 

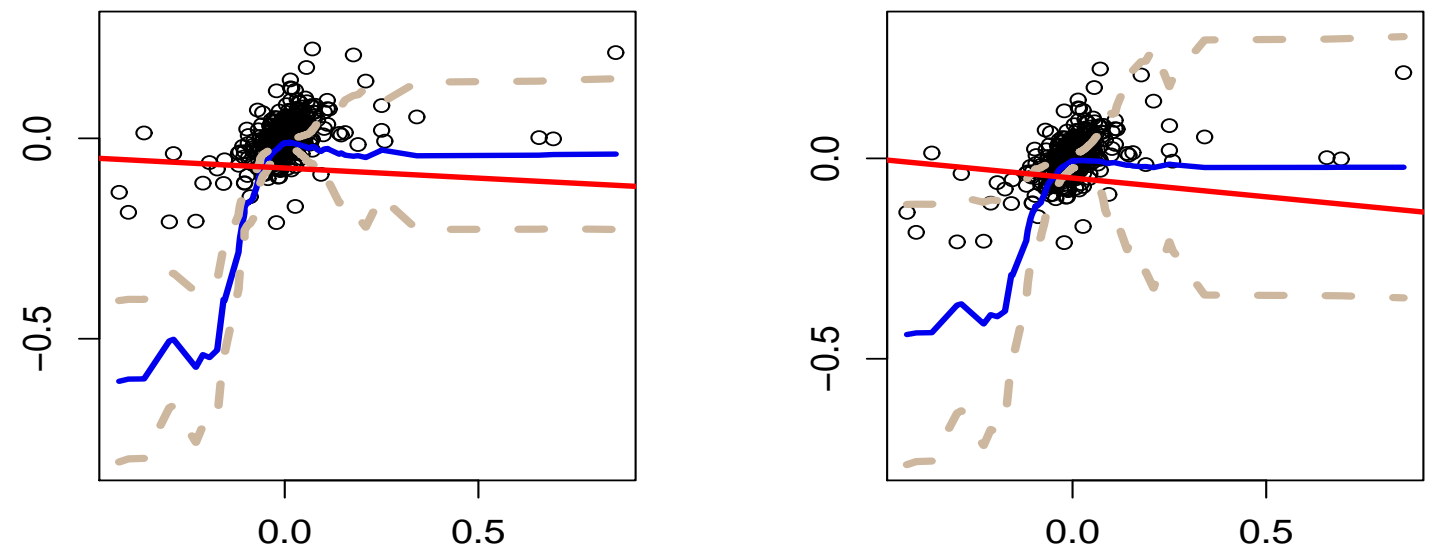

Figure 1.1: Goldman Sachs (GS) and Citigroup (C) weekly returns 0.05(left) and 0.1(right) quantile functions. The $y$-axis is GS daily returns and the $x$-axis is the $\mathrm{C}$ daily returns. The blue curve are the locally linear quantile regression curves (see Appendix A). The locally linear quantile regression bandwidth are 0.1026 and 0.0942 . The red lines are the linear parametric quantile regression line. The antique white dashed curves are the asymptotic confidence band (see Section B) with significance level 0.05. The sample size $N=546$.

quantile, but the challenge for using such models is the curse of dimensionality, as the quantile regression in CoVaR modeling often involves many variables. Thus, we resort to semiparametric partial linear model (PLM) which preserves some flexibility of the nonparametric model while suffers little from the curse of dimensionality.

As an illustration, the VaR/CoVaR of Goldman Sachs (GS) returns are shown, given the returns of Citigroup (C) and S\&P500 (SP). S\&P500 index return is used as a proxy for the market portfolio return.

Choosing market variables is crucial for the VaR/CoVaR estimation. For the variables representing market states, we follow the most popular choices such as VIX, short term liquidity spread, etc. In particular, the variable we use for real estate companies, is the Dow Jones U.S. real estate index. The data is in daily frequency and spans from August 4, 2006 to August 4, 2011.

To see if the estimated VaRs/CoVaRs are accurate, we utilize the backtesting procedures described in Berkowitz et al. (2009). We compare three (Co)VaR estimating methods in this study: VaR computed by linear quantile regression on market variables; CoVaR; PLM CoVaR proposed here. The VaR is one-sided interval prediction, the violations (the asset return exceeds estimated $\mathrm{VaR} / \mathrm{CoVaR}$ ) should happen unpredictably if the VaR algorithm is accurate. In other words, the null hypothesis is that the series of violations of $\mathrm{VaR}$ is a martingale difference given all the past information. Furthermore, if the time series is autocorrelated, we can reject the null hypothesis of martingale difference right away; therefore, autocorrelation tests can be utilized in this context. The Ljung-Box test is not the most appropriate approach here since it has a too strong null hypothesis (i.i.d. sequence). Thus, we additionally apply the Lobato test. The CaViaR test, which is inspired by the CaViaR model, is proposed and shown to have the best overall performance 
by Berkowitz et al. (2009) among other alternative tests with an exclusive desk-level data set. To illustrate the VaR/CoVaR performances in the crisis time, we separately apply the CaViaR test to the violations of the whole sample period and to the financial crisis period.

The results show that for the PLM CoVaR of GS given C performs better than the AB and PLM CoVaR given SP during the financial crisis period from mid 2008 to mid 2009. The nonlinearity between GS and C returns may convey information which is incapable to be reflected in the market returns, especially during unstable market conditions.

In contrast to $\Delta \mathrm{CoVaR}$, we use a mathematically more intuitive way to analyze the marginal effect by taking the first order derivative of the quantile function. We call it "marginal contribution of risk" (MCR). Bae et al. (2003) and many others have pointed out the phenomenon of financial contagion across national borders. This motivates us to consider the stock indices of a few developed markets and explore their risk contribution to the global stock market. MCR results show that when the global market condition varies, the source of global market risk can be different. To be more specific, when the global market return is bad, the risk contribution from the U.S. is the largest. On the other hand, during financially stable periods, Hong Kong and Japan are more significant risk contributors than the U.S. to the global market.

This study is organized as follows: Section 2 introduces the construction and the estimation of the PLM model of CoVaR. The backtesting methods and our risk contribution measure are also introduced in this section. Section 3 presents the Goldman Sachs CoVaR time series and the backtesting procedure results. Section 4 presents the conclusion and possible further studies. Appendices describe the detailed estimation and statistical inference procedures used in this study.

\section{Methodology}

Quantile regression is a well-established technique to estimate the conditional quantile function. Koenker and Bassett (1978) focus on the linear functional form. An extension of linear quantile regression is the PLM quantile regression. A partial linear model for the dynamics of assets return quantile is constructed in this section. The construction is justified by a linearity test based on a conservative uniform confidence band proposed in Härdle and Song (2010). For more details on semiparametric modeling and PLM, we refer to Härdle et al. (2004) and Härdle et al. (2000).

The backtesting procedure is done via the CaViaR test. Finally, the methodology of MCR is introduced, which is an intuitive marginal risk contribution measure. We will apply the method to a data set of global market indices in developed countries.

\subsection{Constructing Partial Linear Model (PLM) for CoVaR}

Recall how the CoVaR is constructed:

$$
\begin{aligned}
& \widehat{V a R}_{i, t}=\hat{\alpha}_{i}+\hat{\gamma}_{i} M_{t-1}, \\
& \widehat{C o V a}_{j \mid i, t}^{A B}=\hat{\alpha}_{j \mid i}+\hat{\beta}_{j \mid i} \widehat{V a R}_{i, t}+\hat{\gamma}_{j \mid i}^{\top} M_{t-1} .
\end{aligned}
$$

where $\left(\hat{\alpha}_{i}, \hat{\gamma}_{i}\right)$ and $\left(\hat{\alpha}_{j \mid i}, \hat{\beta}_{j \mid i}, \hat{\gamma}_{j \mid i}\right)$ are estimated from a linear model using standard linear quantile regression. 
We have motivated the need for more general functional forms for the quantile curve. We therefore relax the model to a non- or semiparametric model. The market variable $M_{t}$ is multidimensional and the data frequency here is daily. The following key variables are entering our analysis:

1. VIX: Measuring the model-free implied volatility of the market. This index is known as the "fear gauge" of investors. The historical data can be found on the Chicago Board Options Exchange's website.

2. Short term liquidity spread: Measuring short-term liquidity risk by the difference between the three-month treasury repo rate and the three-month treasury bill rate. The repo data is from the Bloomberg database and the treasury bill rate data is from the Federal Reserve Board H.15.

3. The daily change in the three-month treasury bill rate: AB find that the changes have better explanatory power than the levels for the negative tail behavior of asset returns.

4. The change in the slope of the yield curve: The slope is defined by the difference of the ten-year treasury rate from the three-month treasury bill rate.

5. The change in the credit spread between 10 years BAA-rated bonds and the 10 years treasury rate.

6. The daily Dow Jones U.S. Real Estate index returns: The index reflects the information of lease rates, vacancies, property development and transactions of real estates in the U.S.

7. The daily S\&P500 index returns: The approximate of the theoretical market portfolio returns.

The variables 3, 4, 5 are from the Federal Reserve Board H.15 and the data of 6 and 7 are from Yahoo Finance.

First we conduct a statistical check of the linearity between GS return and the market variables using the confidence band as constructed in Appendix B. As shown in Figure 2.1 (a) and 2.1 (b), except for some ignorable outsiders, the linear quantile regression line lies in the LLQR asymptotic confidence band.

On the other hand, there is nonlinearity between two individual assets $X_{i}$ and $X_{j}$. To illustrate this, we regress $X_{j}$ on $M_{t}$, and then take the residuals and regress them on $X_{i}$. Again the $X_{j, t}$ is GS daily return and $X_{i}$ is $\mathrm{C}$ daily return. The result is shown in Figure 2.2. The linear QR line (red) lies well outside the LLQR confidence band (magenta) when the $\mathrm{C}$ return is negative. The linear quantile regression line is fairly flat. The risk of using a linear model is obvious in this figure: the linear regression can "average out" the humped relation of the underlying structure (blue), and therefore imply a model risk in estimation.

Based on the results of the linearity tests above, we construct a PLM model:

$$
\begin{aligned}
& X_{i, t}=\alpha_{i}+\gamma_{i}^{\top} M_{t-1}+\varepsilon_{i, t}, \\
& X_{j, t}=\tilde{\alpha}_{j \mid i}+\tilde{\beta}_{j \mid i}^{\top} M_{t-1}+l_{j \mid i}\left(X_{i, t}\right)+\varepsilon_{j, t},
\end{aligned}
$$



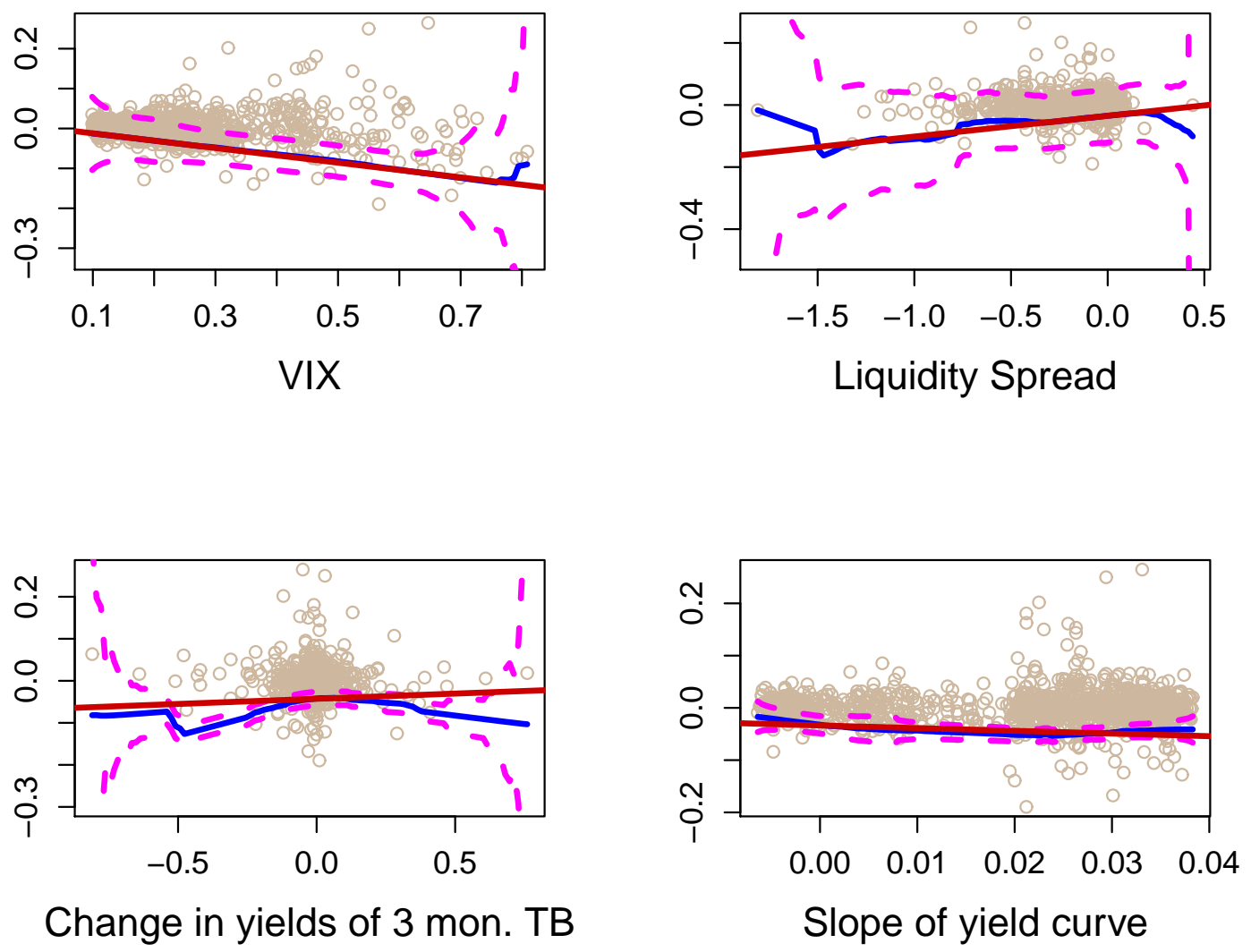

(a)

Figure 2.1 

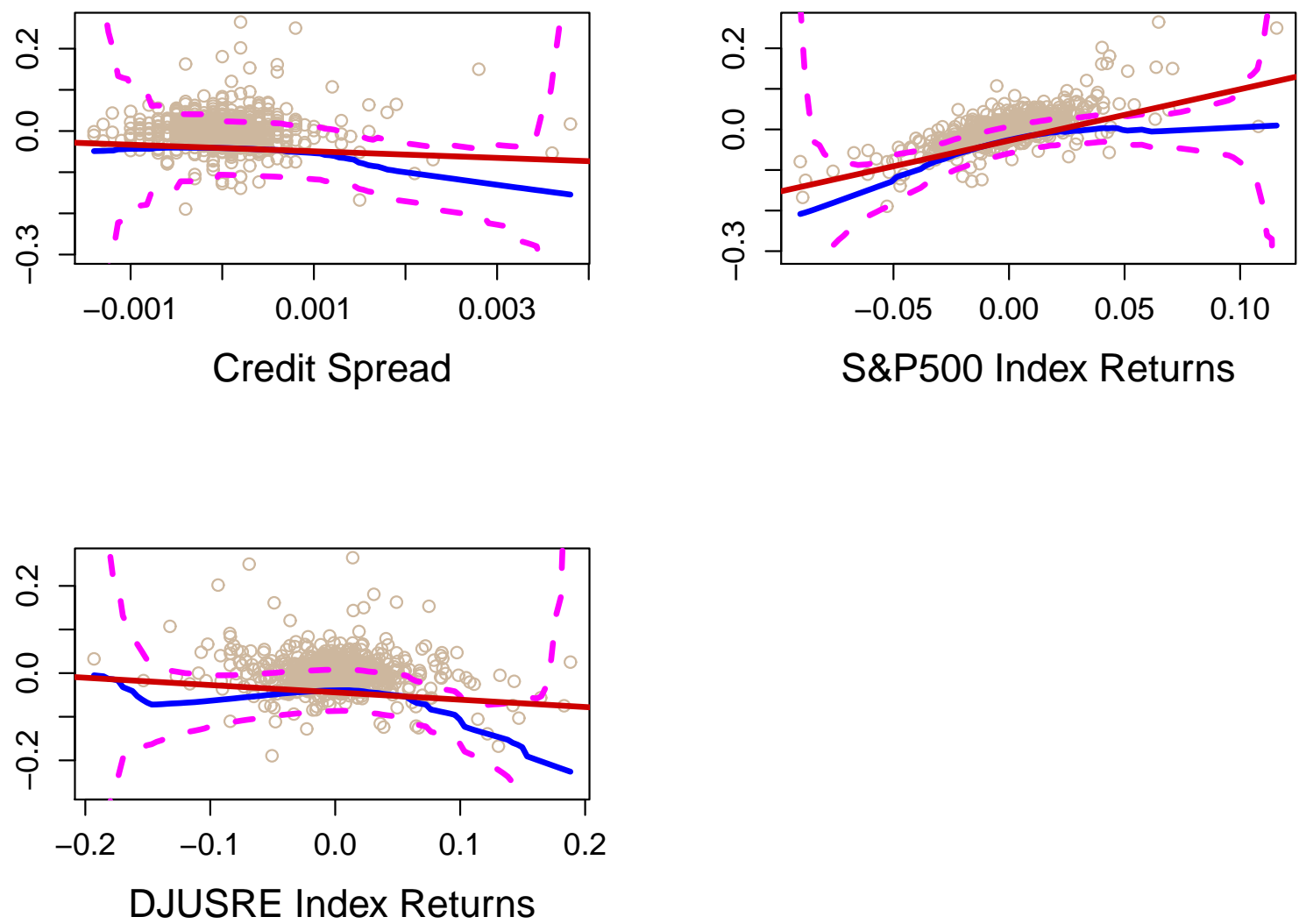

(b)

Figure 2.1: The scatter plots of GS daily returns to the 7 market variables with the LLQR curves. The bandwidths are selected by the method described in Appendix A. The LLQR bandwidths are $0.1101,0.1668,0.2449,0.0053,0.0088,0.0295$ and 0.0569 . The data period is from August 4, 2006 to August 4, 2011. $N=1260 . \tau=0.05$. 


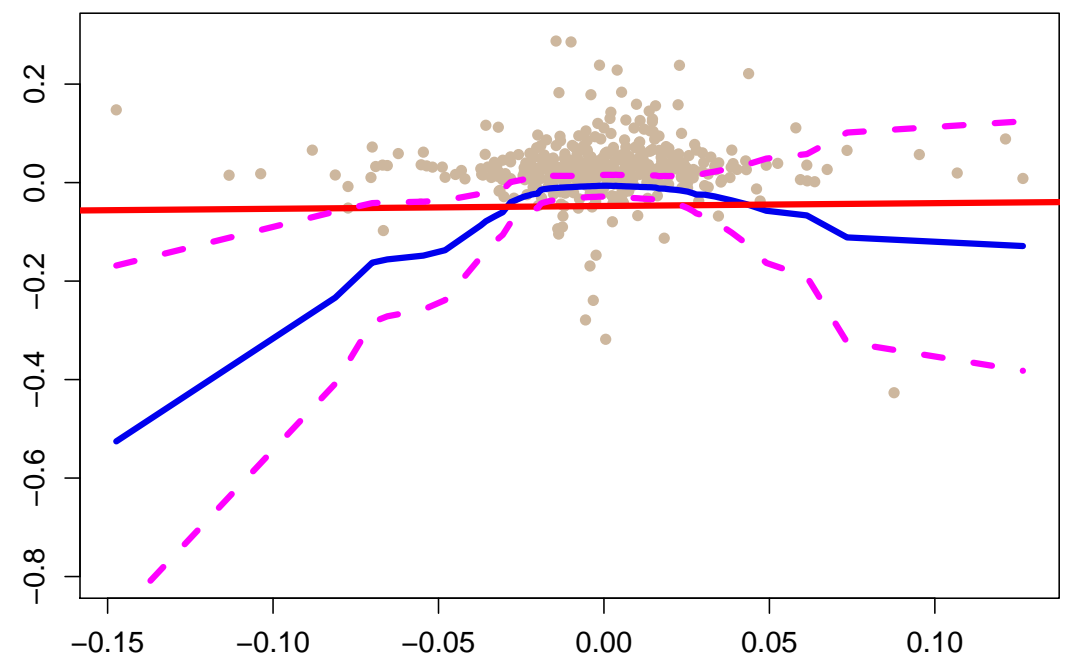

Figure 2.2: The nonparametric part $\hat{l}_{G S \mid C}(\cdot)$ of the PLM estimation. The $y$-axis is the GS daily returns. The $x$-axis is the $\mathrm{C}$ daily returns. The blue curve is the LLQR quantile curve. The red line is the linear parametric quantile line. The magenta dashed curves are the asymptotic confidence band with significance level 0.05. The data is from June 25, 2008 to December 23, 2009. 378 observations. Bandwidth $=0.1255 . \tau=0.05$.

where $X_{i, t}, X_{j, t}$ are asset returns of $i, j$ firms. $M_{t}$ is a vector of market variables at time $t$ as introduced before. If $i=\mathrm{S} \& \mathrm{P} 500, M_{t}$ is set to consist of the first 6 market variables only. Notice the variable $X_{i, t}$ enter the equation (8) nonlinearly.

Applying the algorithm of Koenker and Bassett (1978) to (7) and the process described in Appendix C to equation (8), we get $\left\{\hat{\alpha}_{i}, \hat{\gamma}_{i}\right\}$ and $\left\{\hat{\alpha}_{j \mid i}, \hat{\beta}_{i}, \hat{l}(\cdot)\right\}$ with $F_{\varepsilon_{i, t}}^{-1}\left(\tau \mid M_{t-1}\right)=0$ for (7) and $F_{\varepsilon_{j, t}}^{-1}\left(\tau \mid M_{t-1}, X_{i, t}\right)=0$ for (8). Finally, we estimate the PLM $C o V a R_{j \mid i, t}$ by

$$
\begin{aligned}
& \widehat{V a R}_{i, t}=\hat{\alpha}_{i}+\hat{\gamma}_{i}^{\top} M_{t-1} \\
& \widehat{C o V a R_{j \mid i, t}^{P L M}}=\hat{\tilde{\alpha}}_{j \mid i}+\hat{\tilde{\beta}}_{j}^{\top} M_{t-1}+\hat{l}_{j \mid i}\left(\widehat{V G R}_{i, t}\right) .
\end{aligned}
$$

\subsection{Backtesting}

The goal of the backtesting procedure is to check if the VaR/CoVaR is accurate enough so that managerial decisions can be made based on them. The VaR forecast is a (one-sided) interval forecast. If the VaR algorithm is correct, then the violations should be unpredictable, after using all the past information. Formally, if we define the violation time series as

$$
I_{t}= \begin{cases}1, & \text { if } X_{t}<\widehat{\operatorname{VaR} \tau} \\ 0, & \text { otherwise }\end{cases}
$$

Where $\widehat{\operatorname{VaR}_{t}^{\tau}}$ can be replaced by $\widehat{C o V a R}{ }_{t}^{\tau}$ in the case of CoVaR. I should form a sequence of martingale difference. 
There is a large literature on martingale difference tests. We adopt Ljung-Box test, Lobato test and the CaViaR test. The Ljung-Box test and Lobato test aim to check whether the time series is autocorrelated. If the time series is autocorrelated, then we reject of course the hypothesis that the time series is a martingale difference.

Particularly, let $\hat{\rho}_{k}$ be the estimated autocorrelation of lag $k$ of the sequence of violation $\left\{I_{t}\right\}$ and $n$ be the length of the time series. The Ljung-Box test statistics is:

$$
\mathrm{LB}(m)=n(n+2) \sum_{k=1}^{m} \frac{\hat{\rho}_{k}^{2}}{n-k} \stackrel{\mathcal{L}}{\rightarrow} \chi(m),
$$

as $n \rightarrow \infty$.

This test is too strong though in the sense that the asymptotic distribution is derived based on the i.i.d. assumption. A modified Box-Pierce test is proposed by Lobato et al. (2001), who also consider the test of no autocorrelation, but their test is more robust to the correlation of higher (greater than the first) moments. (Autocorrelation in higher moments does not contradict with the martingale difference hypothesis.) The test statistics is given by

$$
\mathrm{L}(m)=n \sum_{k=1}^{m} \frac{\hat{\rho}_{k}^{2}}{\hat{v}_{k k}} \stackrel{\mathcal{L}}{\rightarrow} \chi(m),
$$

as $n \rightarrow \infty$, where

$$
\hat{v}_{k k}=\frac{\frac{1}{n} \sum_{i=1}^{n-k}\left(y_{i}-\bar{y}\right)^{2}\left(y_{i+k}-\bar{y}\right)^{2}}{\left\{\frac{1}{N} \sum_{i=1}^{n}\left(y_{i}-\bar{y}\right)^{2}\right\}^{2}} .
$$

The CaViaR test, proposed by Berkowitz et al. (2009), is based on the idea that if the sequence of violation is a martingale difference, there ought to be no correlation between any function of the past variables and the current violation. One way to test this uncorrelatedness is through a linear model. The model is:

$$
I_{t}=\alpha+\beta_{1} I_{t-1}+\beta_{2} V a R_{t}+u_{t},
$$

where $V a R_{t}$ can be replaced by $\mathrm{CoVa} R_{t}$ in the case of conditional VaR. The residual $u_{t}$ follows a Logistic distribution since $I_{t}$ is binary. We get the estimates of the coefficients $\left(\hat{\beta}_{1}, \hat{\beta}_{2}\right)^{\top}$. Therefore the null hypothesis is $\hat{\beta}_{1}=\hat{\beta}_{2}=0$. This hypothesis can be tested by Wald's test.

We set $m=1$ or 5 for the Ljung-Box and Lobato tests. For the CaViaR test, two data periods are considered separately. The first is the overall data from August 4, 2006 to August 4, 2011. The second is the data from August 4, 2008 to August 4, 2009, the period when the financial market reached its bottom. By separately testing the two periods, we can gain more insights into the PLM model.

\subsection{Risk Contribution Measure}

The risk contribution of one firm to the market is one of the top concerns among central bankers. The regulator can restrict the risky behaviors of the financial institution with high risk contribution to the market, and reduce the institution's incentive to take more risk. AB propose the idea of 
$\Delta \mathrm{CoVaR}$, which is defined by

$$
\Delta \operatorname{CoVaR} R_{j \mid i, t}^{\tau}=\operatorname{CoVa} R_{j \mid i, t}^{\tau}-\operatorname{CoVa} R_{j \mid i, t}^{0.5}
$$

where $\operatorname{CoVaR}_{j \mid i, t}^{\tau}$ is defined as in the introduction. $j, i$ represent the financial system and an individual asset. $\tau=0.5$ corresponds to the normal state of the individual asset $i$. This is essentially a sensitivity measure quantifying the effect to the financial system from the occurrence of a tail event of asset $X_{i}$.

In this study we adopt a mathematically intuitive way to measure the marginal effect by searching the first order derivative of the quantile function. Because the spillover effect from stock market to stock market has already got much attention, it is important to investigate the risk contribution of a local market to the global stock market. The estimation is conducted as follows:

First, one estimates the following model nonparametrically:

$$
X_{j, t}=f_{j}^{0.05}\left(X_{t}\right)+\varepsilon_{j}
$$

The quantile function $f_{j}^{0.05}(\cdot)$ is estimated with local linear quantile regression with $\tau=0.05$, described with more details in Appendix A. $X_{j}$ is the weekly return of the stock index of an individual country and $X$ is the weekly return of the global stock market.

Second, with $\hat{f}_{j}^{0.05}(\cdot)$, we compute the "marginal contribution of risk" (MCR) of institution $j$ by

$$
M C R_{j}^{\tau}=\left.\frac{\partial \hat{f}_{j}^{0.05}(x)}{\partial x}\right|_{x=\hat{F}_{X}^{-1}\left(\tau_{k}\right)}
$$

where $\hat{F}^{-1}\left(\tau_{k}\right)$ is a consistent estimator of the $\tau_{k}$ quantile of the global market return, and it can be estimated by regressing $X_{t}$ on the time trend. We put $k=1,2$ with $\tau_{1}=0.5$ and $\tau_{2}=0.05$. The quantity (14) is similar to the MES proposed by Acharya et al. (2010) in the sense that the conditioned event belongs to the information set of the market return, but we reformulate it in the VaR framework instead of the expected shortfall framework.

There are some properties of the $M C R$ to be described further. First, $\tau_{k}$ determines the condition of the global stock market. This allows us to explore the risk contribution from the index $j$ to the global market given different global market status. Second, the higher the value of MCR, the more risk factor $j$ imposes on the market in terms of risk. Third, since the function $f_{j}^{0.05}(\cdot)$ is estimated by LLQR, the quantile curve is locally linear, and therefore the local first order derivative is straightforward to compute.

We choose indices $j=$ S\&P500, NIKKEI225, FTSE100, DAX30, CAC40, Heng Seng as the approximate of the market returns of each developed country or market. The global market is approximated by the MSCI World (developed countries) market index. The data is weekly from April 11, 2004 to April 11, 2011 and $\tau=0.05$ 


\section{Results}

\subsection{CoVaR Estimation}

The estimation results of $\mathrm{VaR} / \mathrm{CoVaR}$ are shown in this section. We compute three types of $\mathrm{VaR} / \mathrm{CoVaR}$ of GS, with a moving window size of 126 business days and $\tau=0.05$.

First, the VaR of GS is estimated:

$$
\widehat{V a R}_{G S, t}=\hat{\alpha}_{G S}+\hat{\gamma}_{G S}^{\top} M_{t-1}
$$

using linear quantile regression, and $M_{t} \in \mathbb{R}^{7}$ is introduced in Section 2.1.

Second, the CoVaR of GS given C returns is estimated:

$$
\begin{aligned}
& \widehat{\operatorname{VaR}}_{C, t}=\hat{\alpha}_{C}+\hat{\gamma}_{C}^{\top} M_{t-1} \\
& \widehat{\operatorname{CoVa} R}_{G S \mid C, t}^{A B}=\hat{\alpha}_{G S \mid C}+\hat{\beta}_{G S \mid C} \widehat{V a R}_{C, t}+\hat{\gamma}_{G S \mid C}^{\top} M_{t-1} .
\end{aligned}
$$

If the $\mathrm{SP}$ replaces $\mathrm{C}$, the estimates are generated from

$$
\begin{aligned}
& \widehat{V a R}_{S P, t}=\hat{\alpha}_{S P}+\hat{\gamma}_{S P}^{\top} \widetilde{M}_{t-1} \\
& \widehat{C o V a R}_{G S \mid S P, t}^{A B}=\hat{\alpha}_{G S \mid S P}+\hat{\beta}_{G S \mid S P} \widehat{V a R}_{S P, t}+\hat{\gamma}_{G S \mid S P}^{\top} \widetilde{M}_{t-1},
\end{aligned}
$$

where $\widetilde{M}_{t} \in \mathbb{R}^{6}$ is the vector of market variables without the market portfolio return.

Third, the PLM CoVaR is generated:

$$
\begin{aligned}
& \widehat{V a R}_{C, t}=\hat{\alpha}_{C}+\hat{\gamma}_{C}^{\top} M_{t-1} ; \\
& \widehat{\operatorname{CoVa} R} P_{G S \mid C, t}=\hat{\tilde{\alpha}}_{G S \mid C}+\hat{\tilde{\beta}}_{G S \mid C}^{\top} M_{t-1}+\hat{l}_{G S \mid C}\left(\widehat{V a R}_{C, t}\right) .
\end{aligned}
$$

If SP replaces C:

$$
\begin{aligned}
& \widehat{V a R}_{S P, t}=\hat{\alpha}_{S P}+\hat{\gamma}_{S P}^{\top} \widetilde{M}_{t-1} ; \\
& \widehat{C o V a R}_{G S \mid S P, t}^{P L M}=\hat{\tilde{\alpha}}_{G S \mid S P}+\hat{\tilde{\beta}}_{G S \mid S P}^{\top} \widetilde{M}_{t-1}+\hat{l}_{G S \mid S P}\left(\widehat{V G R}_{S P, t}\right) .
\end{aligned}
$$

The coefficients in (15), (16), (17), (18), (19), (20) and (22) are estimated from the linear quantile regression and those in (21) and (23) are estimated from the method described in Appendix C.

Figure 3.1 shows the $\widehat{V a R}_{G S, t}$ sequence. The VaR forecasts (red) seem to form a lower cover of the GS returns (blue). This suggests that the market variables $M_{t}$ have some predictive power for the left tail quantile of the GS return distribution. Figure 3.2 shows the sequences $\widehat{C o V a R} A B S A P, t$ (cyan) and $\widehat{C o V a} R_{G S \mid C, t}^{P L M}$ (light green). As the time series of the estimates is too volatile, we smooth it further by the median LLQR. The two estimates are similar as the market state is stable, but during the period of financial instability (from mid 2008 to mid 2009), the two estimates have different behavior. The performance of these estimates are evaluated by backtesting procedure in Section 3.2 .

Table 3.1 shows the summary statistics of the VaR/CoVaR estimates. The first three rows show 


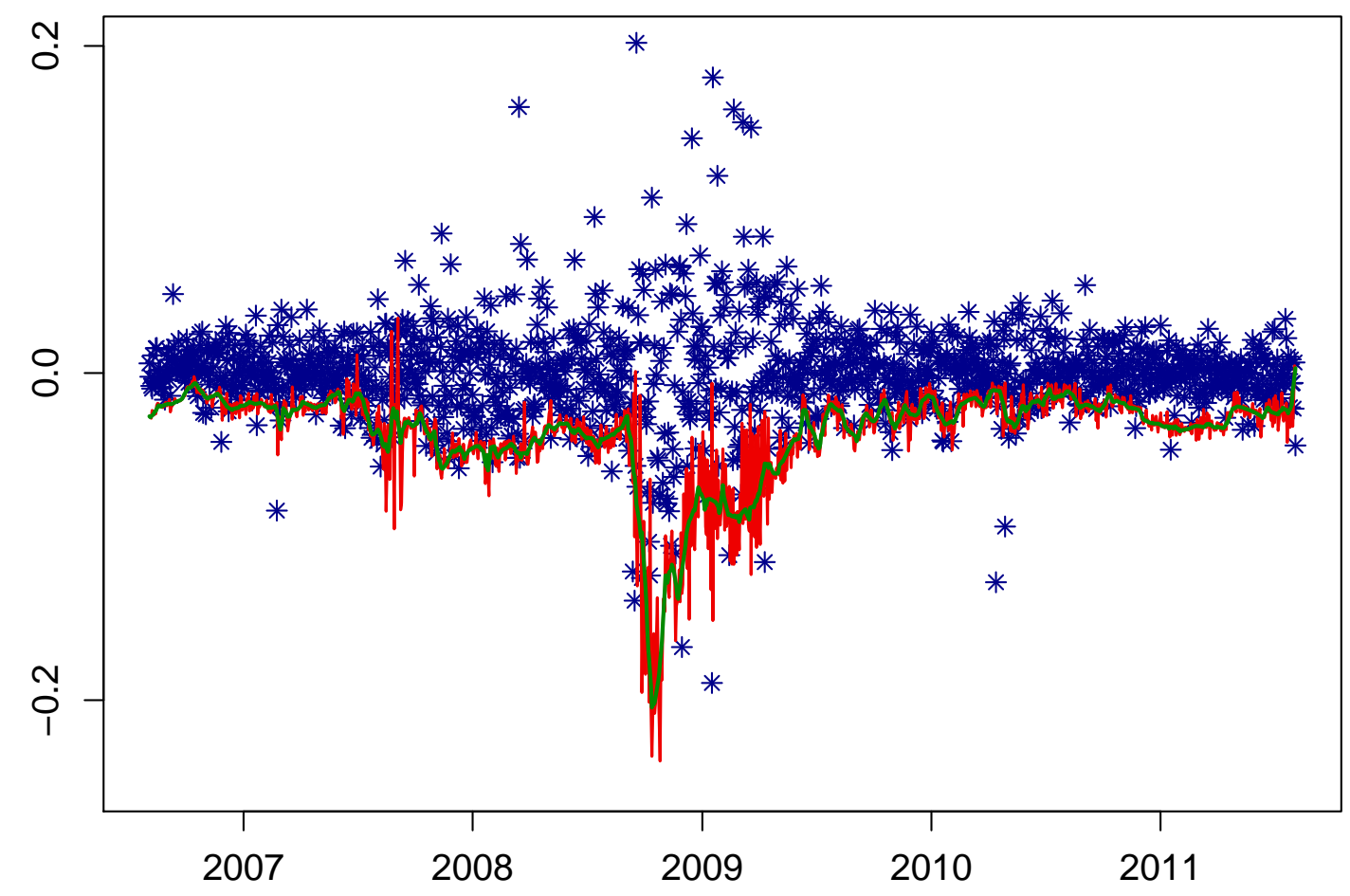

Figure 3.1: The $\widehat{V a R}_{G S, t}$. The red line is the $\widehat{V a R}_{G S, t}$ and blue stars are daily returns of GS. The dark green curve is the meadian smoother of the $\widehat{V a R}_{G S, t}$ curve with $\mathrm{h}=2.75 . \tau=0.05$. The window size is 252 days.

the summary statistics of $\widehat{V a R}_{G S, t}, \widehat{V a R}_{C, t}$ and $\widehat{V a R}_{S P, t}$. The $\widehat{V a R}_{G S, t}$ has lower mean and higher standard deviation than the other two. Particularly during 2008 to 2009, the standard deviation of the GS VaR is twice as much as the other two. The mean and standard deviation of the $\widehat{V a R}_{C, t}$ and $\widehat{V a R}_{S P, t}$ are rather similar. The last four rows show the summary statistics of $\widehat{C o V a R} \widehat{R}_{G S \mid C, t}$, $\widehat{C o V a R}_{G S \mid C, t}^{A B}, \widehat{C o V a} R_{G S \mid S P, t}^{P L M}$ and $\widehat{C o V a} R_{G S \mid S P, t}^{A B}$. This shows that the CoVaR obtaining from the $\mathrm{AB}$ model has smaller mean but greater standard deviation than the CoVaR obtaining from PLM model.

Figure 3.3 shows the bandwidth sequence of the nonparametric part of the PLM estimation. The bandwidth varies with time. Before mid 2007, the bandwidth sequence is stably jumping around 0.2. After that the sequence becomes very volatile. This may have something to do with the rising systemic risk. 


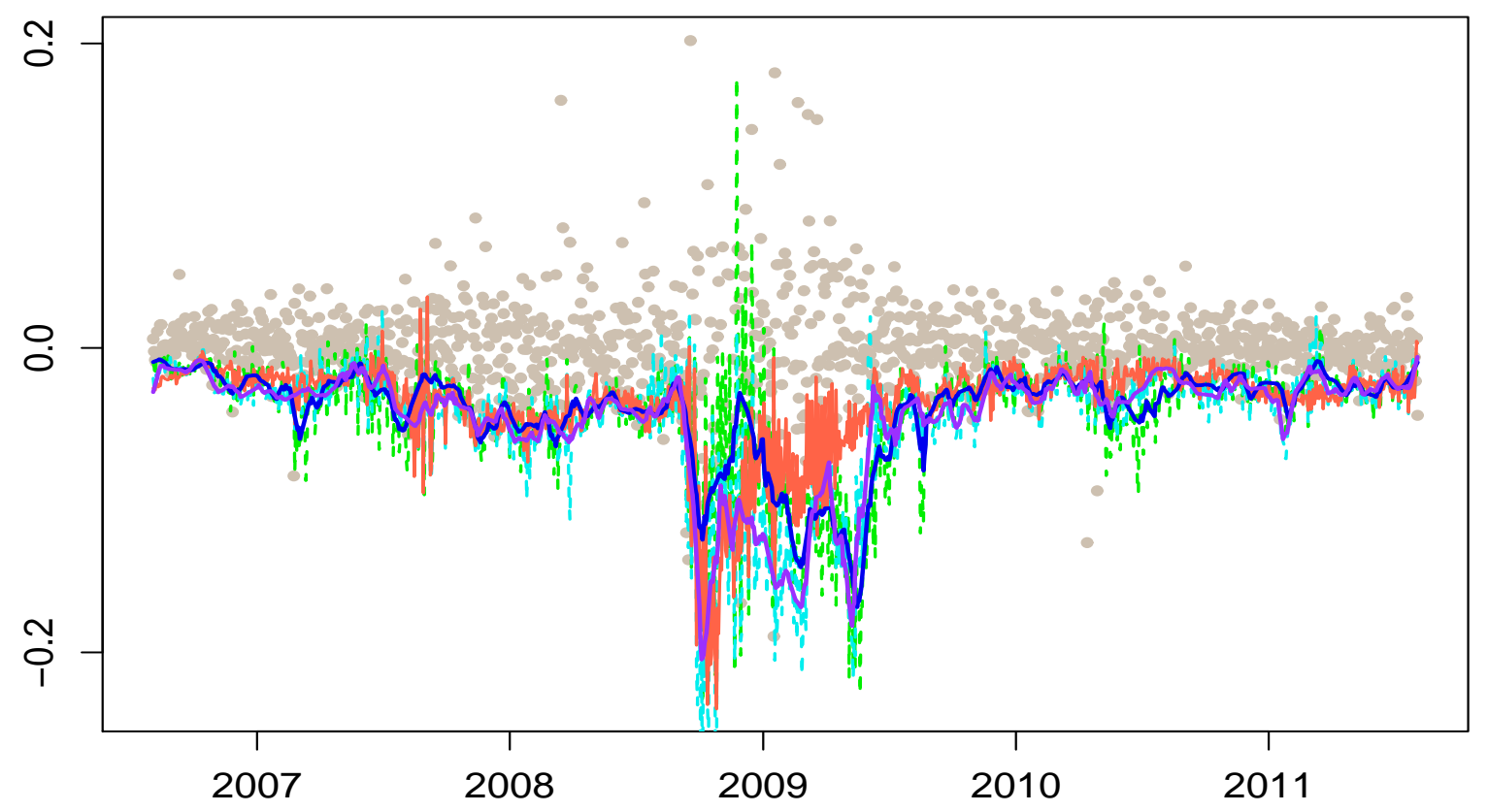

Figure 3.2: The CoVaR of GS given the VaR of C. The gray dots are daily returns of GS. The light green dashed curve is the $\widehat{C o V a R} R_{G S \mid C, t}$. The blue curve is the median LLQR smoother of the light green dashed curve with $h=3.19$. The cyan dashed curve is the $\widehat{C o V a} R_{G S \mid C, t}$. The purple curve is the median LLQR smoother of the cyan dashed curve with $h=3.90$. The red curve is the $\widehat{V a R}_{G S, t} \cdot \tau=0.05$. The moving window size is 126 days. 


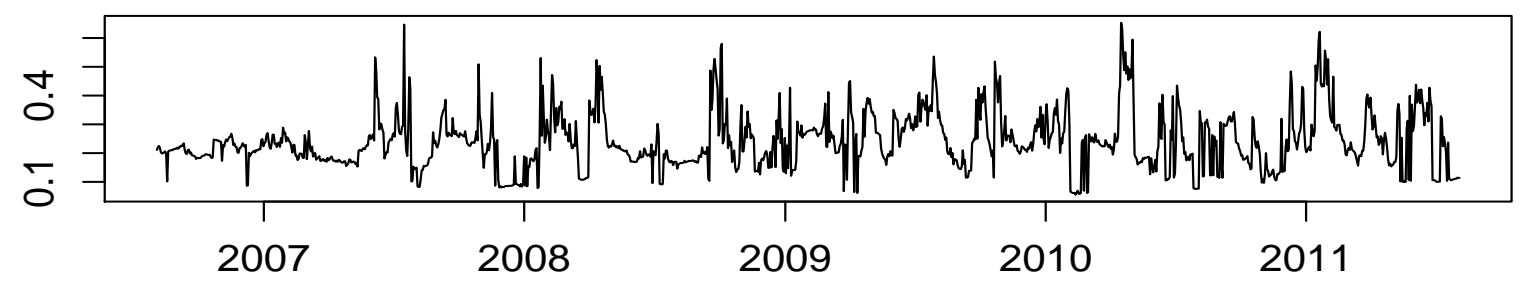

Figure 3.3: LLQR bandwidth in the daily estimation of $\widehat{C o V a} R_{G S \mid C, t}^{P L M}$. The average bandwidth is 0.24 .

Table 3.1: VaR/CoVaR summary statistics. The overall period is from August 4, 2006 to August 4, 2011. The crisis period is from August 4, 2008 to August 4, 2009. The numbers in the table are scaled up by $10^{2}$.

\begin{tabular}{|c|c|c|c|c|}
\hline & mean-overall & sd-overall & mean-crisis & sd-crisis \\
\hline$\widehat{\operatorname{VaR}}_{G S, t}$ & -3.66 & 3.08 & -7.43 & 4.76 \\
\hline$\widehat{\operatorname{VaR}}_{C, t}$ & -2.63 & 1.67 & -4.62 & 2.25 \\
\hline$\widehat{V a R}_{S P, t}$ & -2.09 & 1.57 & -3.88 & 2.24 \\
\hline$\widehat{\operatorname{CoVaR}}_{G S \mid C, t}^{P L M}$ & -4.26 & 3.84 & -8.79 & 5.97 \\
\hline$\widehat{\operatorname{CoVa}}_{G S \mid C, t}^{A B}$ & -4.60 & 4.30 & -10.36 & 6.32 \\
\hline$\widehat{C o V a R} R_{G S \mid S P, t}^{P L M}$ & -3.86 & 3.30 & -8.20 & 4.69 \\
\hline$\widehat{\operatorname{CoVaR}}_{G S \mid S P, t}^{A B}$ & -5.81 & 4.56 & -12.65 & 5.56 \\
\hline
\end{tabular}

\subsection{Backtesting}

For the evaluation of the CoVaR models, we resort to the backtesting procedure described in Section 2.2. In order to perform the backtesting procedure, the sequences $\left\{I_{t}\right\}$ (defined in Section 2.2) have to be computed for all VaR/CoVaR estimates. Figure 3.4 shows the timings of the violations $\left\{t: I_{t}=1\right\}$ of $\widehat{C o V a R}_{G S \mid C, t}^{P L M}, \widehat{C o V a}_{G S \mid C, t}^{A B}$ and $\widehat{V a R}_{G S, t}$. This figure shows the total number of violations of PLM CoVaR and CoVaR are similar, while $\widehat{\operatorname{VaR}}_{G S, t}$ has more violations than the both. The $\widehat{V a R}_{G S, t}$ has a few clusters of violations in both financial stable and unstable periods. This may result from the failure $\widehat{V a R}_{G S, t}$ to adapt for the negative shocks. The violations of $\widehat{C o V a}_{G S \mid C, t}^{P L M}$ are more evenly distributed. The violations of $\widehat{\mathrm{CoVa} R_{G S \mid C, t}}{ }^{A B}$ have large clusters during financially stable period, while the violation during financial crisis period is meager. This contrast suggests that $\widehat{C o V a} R_{G S \mid C, t}^{A B}$ tend to overreact, as it is slack during the stable period but is too tight during the unstable period.

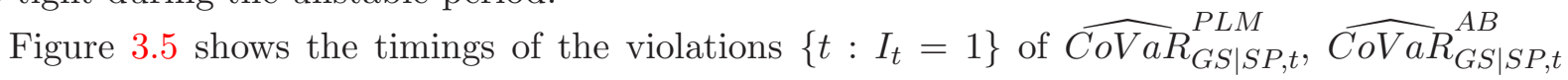


and $\widehat{V a R}_{G S, t}$. The overall number of violations of $\widehat{C o V a R}_{G S \mid S P, t}^{P L M}$ is more than that of $\widehat{V G R}_{G S, t}$, and it has many clusters. $\widehat{C o V a} R_{G S \mid S P, t}^{P L M}$ behaves differently from $\widehat{C o V a} R_{G S \mid C, t}^{P L M}$. The SP may not be more informative than $\mathrm{C}$, though the efficient market hypothesis suggests so. The violation of $\widehat{C o V a R}_{G S \mid S P, t}^{A B}$ is fewer than the other two measures, and the clustering is not significant.

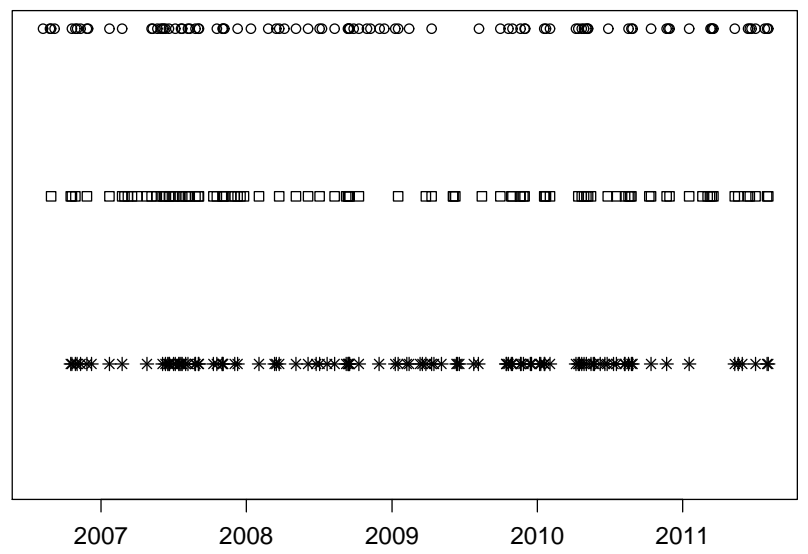

Figure 3.4: The timings of violations $\left\{t: I_{t}=1\right\}$. The top circles are the violations of the $\widehat{C o V a R}_{G S \mid C, t}^{P L M}$, totally 95 violations. The middle squares are the violations of $\widehat{\mathrm{CoVa}_{G S \mid C, t}}{ }^{A B}$, totally 98 violations. The bottom stars are the violations of $\widehat{V a R}_{G S, t}$, totally 109 violations. Overall data $N=1260$.

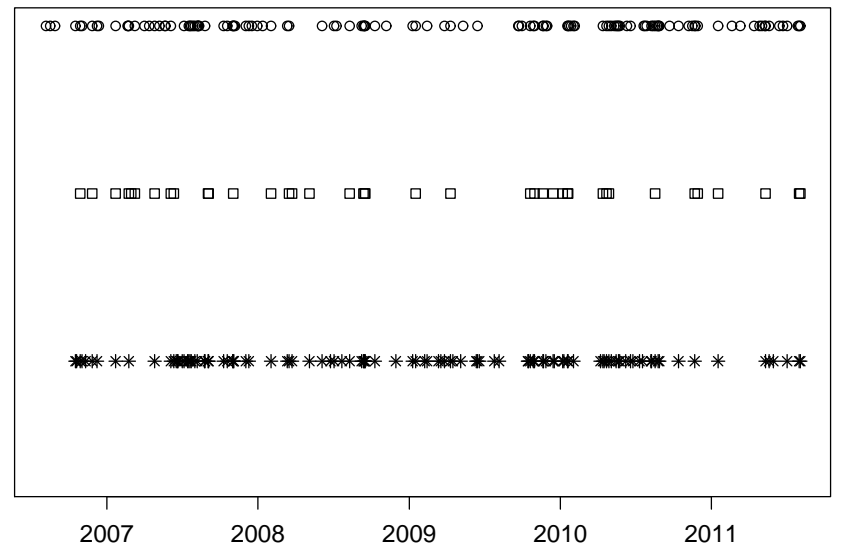

Figure 3.5: The timings of violations $\left\{t: I_{t}=1\right\}$. The top circles are the violations of $\widehat{C o V a R}_{G S \mid S P, t}^{P L M}$, totally 123 violations. The middle squares are the violations of $\widehat{C o V a R}{ }_{G S \mid S P, t}^{A B}$, totally 39 violations. The bottom stars are the violations of $\widehat{V a R}_{G S, t}$, totally 109 violations. Overall data $N=1260$.

The backtesting procedure is performed separately for each sequence of $\left\{I_{t}\right\}$. The null hypothesis is that each sequence $\left\{I_{t}\right\}$ forms a series of martingale difference. Six different tests are applied for 
each $\left\{I_{t}\right\}$ : Ljung-Box tests with lags 1 and 5, Lobato test with lags 1 and 5 and finally the CaViaR test with two data periods: overall and crisis period.

The result is shown in Table 3.2. First, in Panel 1 of Table 3.2, the $\widehat{V a R}_{G S, t}$ is rejected by the $\mathrm{LB}(5)$ test and the two CaViaR tests. This shows that a linear quantile regression on the seven market variables may not give accurate estimates, in the sense that the violation $\left\{I_{t}\right\}$ of $\widehat{V a R}_{G S, t}$ does not form a martingale sequence. Next we turn to the $\widehat{\mathrm{CoVa}^{A} R_{G S \mid S P, t}}$ and $\widehat{C o V a} R_{G S \mid S P, t}^{P L M}$. In Panel 2, the low $p$-values of the two CaViaR tests show that both the AB model and PLM model conditioned on SP are rejected, though the $p$-value of the AB model almost reaches the $5 \%$ significant level. In particular, the $\widehat{\mathrm{CoVa}_{\mathrm{O}}} \mathrm{PLS}_{G S P, t}$ is rejected by the $\mathrm{L}(5)$ and $\mathrm{LB}(5)$ tests. Both the parametric and semiparametric models fail with this choice of variable. This suggests that the market return does not provide enough information in risk measurement.

We therefore need more informative variables. Panel 3 of Table 3.2 illustrates this by using $\mathrm{C}$ daily returns, which may contain information not revealed in the market and improve the performance of the estimates. The $\widehat{C o V a} R_{G S \mid C, t}^{A B}$ is rejected by the two CaViaR tests and the $\mathrm{LB}(1)$ test with $0.1 \%$ and $5 \%$ significant level. However, $\widehat{C o V a R} R_{G S \mid C, t}^{P L M}$ is not rejected by the CaViaR-crisis test. This implies that the nonparametric part in the PLM model captures the nonlinear effect of $\mathrm{C}$ returns to GS returns, which can lead to better risk-measuring performance.

Table 3.2: Goldman Sachs VaR/CoVaR backtesting $p$-values. The overall period is from August 4, 2006 to August 4, 2011. The crisis period is from August 4, 2008 to August 4, 2009. LB(1) and $\mathrm{LB}(5)$ are the Ljung-Box tests of lags 1 and $5 . \mathrm{L}(1)$ and $\mathrm{L}(5)$ are the Lobato tests of lags 1 and 5 . CaViaR-overall and CaViaR-crisis are two CaViaR tests described in Section 2.2 applied on the two data periods.

\begin{tabular}{|c|c|c|c|c|c|c|}
\hline Measure & $\mathrm{LB}(1)$ & $\mathrm{LB}(5)$ & $\mathrm{L}(1)$ & $\mathrm{L}(5)$ & CaViaR-overall & CaViaR-crisis \\
\hline \multicolumn{7}{|c|}{$\underline{\text { Panel } 1}$} \\
\hline$\widehat{\operatorname{VaR}}_{G S, t}$ & 0.3449 & $0.0253^{*}$ & 0.3931 & 0.1310 & $1.265 \times 10^{-6 * * *}$ & $0.0024^{* *}$ \\
\hline \multicolumn{7}{|c|}{$\underline{\text { Panel } 2}$} \\
\hline$\widehat{C o V a}_{G S \mid S P, t}^{A B}$ & 0.0869 & 0.2059 & 0.2684 & 0.6586 & $8.716 \times 10^{-7 * * *}$ & $0.0424^{*}$ \\
\hline$\widehat{\operatorname{CoVa}}_{G S \mid S P, t}^{P L M}$ & 0.0518 & $0.0006^{* * *}$ & 0.0999 & $0.0117^{*}$ & $2.2 \times 10^{-16 * * *}$ & $0.0019^{* *}$ \\
\hline \multicolumn{7}{|c|}{$\underline{\text { Panel } 3}$} \\
\hline$\widehat{\operatorname{CoVa}^{\prime}} R_{G S \mid C, t}^{A B}$ & $0.0489 *$ & 0.2143 & 0.1201 & 0.4335 & $3.378 \times 10^{-9 * * *}$ & $0.0001^{* * *}$ \\
\hline$\widehat{C o V a}_{G S \mid C, t}^{P L M}$ & 0.8109 & $0.0251^{*}$ & 0.8162 & 0.2306 & $2.946 \times 10^{-9 * * *}$ & 0.0535 \\
\hline
\end{tabular}

\subsection{Global Risk Contribution}

In this section we present the $M C R$ (defined in Section 2.3), which measures the marginal risk contribution of risk factors. We choose $\tau_{1}=0.5$, associated to the normal (median) state and $\tau_{2}=0.05$, associated to an negative extreme state. Figure 3.6 shows the $M C R_{j}^{\tau_{1}}$ from local markets $j$ to the global market. When the MSCI World is (hypothetically) at its normal state, one concludes that the Heng Seng in normal times contributes the most to the MSCI World at all times. The NIKKEI225 places second; the contribution from S\&P500 varies most with the time; 


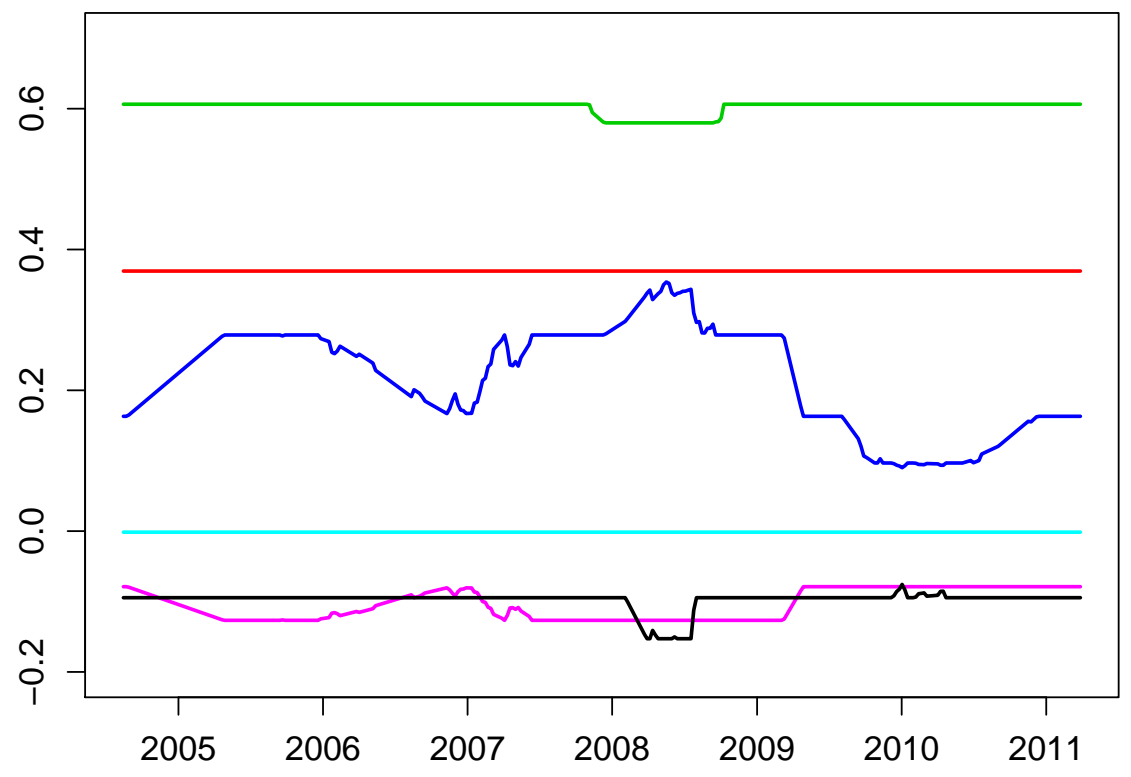

Figure 3.6: The $M C R_{j}^{\tau_{1}}, \tau=0.5 . j$ :CAC, FTSE, DAX, Heng Seng, S\&P500 and NIKKEI225. The global market return is approximated by MSCI World.

the risk contribution from DAX30 is nearly zero. The contribution from CAC40 and FTSE100 are negative.

Assuming that the MSCI World is at its bad state $\left(\tau_{2}=0.05\right)$, the $M C R_{j}^{\tau_{2}}$ differs from $M C R_{j}^{\tau_{1}}$, see Figure 3.7. One sees that the S\&P500 imposes more pressure on the world economy than the other countries. Especially during the financial crisis of 2008 and 2009. The contribution from Heng Seng is no longer of the same significance. The three European markets are relatively stable.

This analysis suggests that the risk contribution from individual stock market varies a lot with the state of global economy.

\section{Conclusion}

In this study we construct a PLM model for the CoVaR, and we compare it to the AB model by backtesting. Results show that PLM CoVaR is preferable especially during a crisis period. The study of the MCR reveals the fact that the risk from each country can vary with the state of global economy.

As an illustration, we only study the Goldman Sachs conditional VaR with Citigroup and S\&P500 as conditioned risk sources. In practice, we need to choose variables. In Hautsch et al. (2011), the Least Absolute Shrinkage and Selection Operator (LASSO) techniques is used to determine the most relevant systemic risk sources from a pool of financial institutions. A VAR (Vector Autoregression) model may be also suitable for capturing the asset dynamics, but the estimation may be more involved. We may include other firm specific variables such as corporate bond yields as these variables can bear other information which is not included in the stock returns or stock 


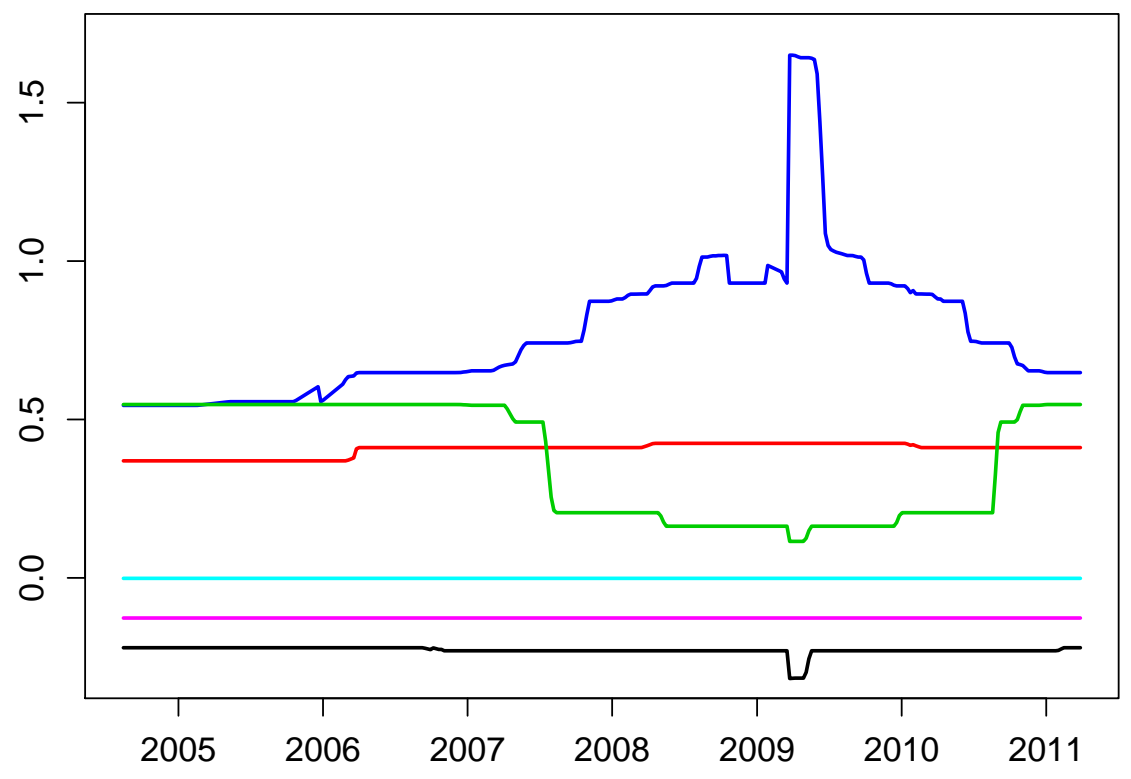

Figure 3.7: The $M C R_{j}^{\tau_{2}}, \tau=0.05 . \quad j$ :CAC, FTSE, DAX, Heng Seng, S\&P500 and NIKKEI225. The global market return is approximated by MSCI World.

indices.

\section{Appendices}

\section{A. Locally Linear Quantile Regression (LLQR)}

Let $\left\{\left(X_{i}, Y_{i}\right)\right\}_{i=1}^{n} \subset \mathbb{R}^{2}$ be i.i.d. bivariate random variables. Denote by $F_{Y \mid x}(u)$ the conditional cdf and $l(x)=F_{Y \mid x}^{-1}(\tau)$ the conditional quantile curve to level $\tau$, given observations $\left\{\left(x_{i}, y_{i}\right)\right\}_{i=1}^{n}$, one may write this as

$$
y_{i}=l\left(x_{i}\right)+\varepsilon_{i},
$$

with $F_{\varepsilon \mid x}^{-1}(\tau)=0$. A locally linear kernel quantile estimator (LLQR) is estimated as $\hat{l}\left(x_{0}\right)=\hat{a}_{0}$ from:

$$
\left(\hat{a}_{0}, \hat{b}_{0}\right)=\underset{\left\{a_{0}, b_{0}\right\}}{\operatorname{argmin}} \sum_{i=1}^{n} K\left(\frac{x_{i}-x_{0}}{h}\right) \rho_{\tau}\left\{y_{i}-a_{0}-b_{0}\left(x_{i}-x_{0}\right)\right\}
$$

where $h$ is the bandwidth, $K(\cdot)$ is a kernel and $\rho_{\tau}(\cdot)$ is the check function given by

$$
\rho_{\tau}(u)=\left(\tau-\mathbf{1}_{\{u<0\}}\right) u
$$


Figure A.1 illustrates the check functions. Different loss functions give different estimates. $u^{2}$ corresponds to the conditional mean. $\rho_{\tau}(u)$ corresponds to the conditional $\tau$ th quantile.

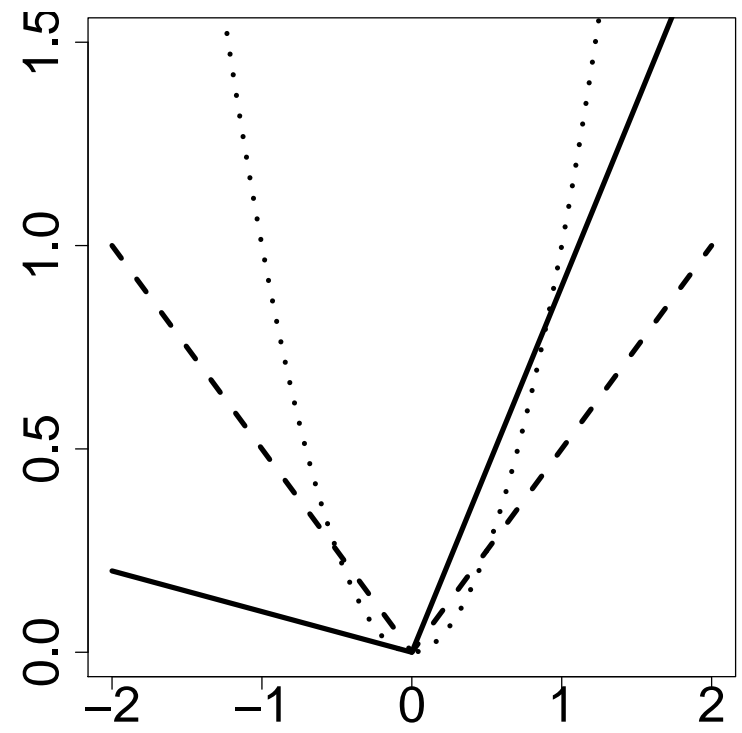

Figure A.1: This figure presents the check function. The dotted line is $u^{2}$. The dashed and solid lines are check functions $\rho_{\tau}(u)$ with $\tau=0.5$ and 0.9 respectively.

It is shown by Fan et al. (1994) that the locally linear kernel estimator is asymptotically efficient in a minimax sense. It also possesses good finite sampling property which is adaptive to a variety of empirical density $g(x)$ and has good boundary property.

Next, we describe the method to compute the bandwidths. The approach used here follows Yu and Jones (1998). The bandwidth is chosen by

$$
h_{\tau}=h_{\text {mean }}\left[\tau(1-\tau) \varphi\left\{\Phi^{-1}(\tau)\right\}^{-2}\right]^{1 / 5},
$$

where $h_{\text {mean }}$ is the locally linear mean regression bandwidth, which can be computed by the algorithm described in Ruppert and Wand (1995) or Ruppert et al. (1995). $\varphi(\cdot)$ and $\Phi(\cdot)$ are the pdf and cdf of the standard normal distribution. Since we discuss the case for VaR, $\tau$ is usually small. $h_{\tau}$ needs to be enlarged to allow for more smoothing (usually taking $1.5 h_{\tau}$ or $2 h_{\tau}$ ).

The approach is acceptable but not so flexible because it is based on assuming the quantile functions are parallel. A more flexible approach was developed by Härdle, Spokoiny and Wang (2011). In order to stabilize the bandwidth choice, we first regress $y_{i}$ on the rank of the corresponding $x_{i}$ and then rescale the resulted estimated values to the original $x$ space. Carroll and Härdle (1989) show that this local bandwidth estimator and the global bandwidth estimator are asymptotically equivalent.

\section{B. Confidence Band for Nonparametric Quantile Estimator}

The uniform confidence band of the quantile estimator is based on the Theorem 2.2 and Corollary 2.1 presented in Härdle and Song (2010). The details are as follows.

Let $\left\{\left(X_{i}, Y_{i}\right)\right\}_{i=1}^{n}$ be as in Appendix A. Define $K_{h}(u)=h^{-1} K(u / h)$ and similar to (24) let $l_{n}(x)$ 

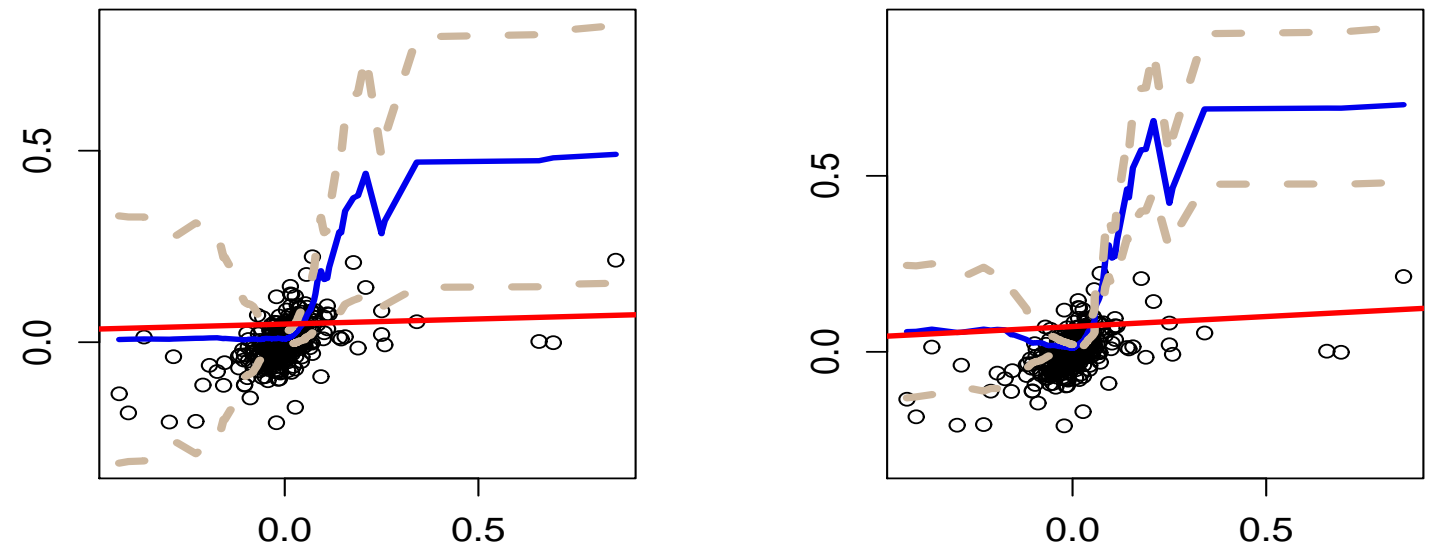

Figure A.2: GS and C weekly returns 0.90 (left) and 0.95(right) quantile functions. The $y$-axis is GS daily returns and the $x$-axis is the $\mathrm{C}$ daily returns. The blue curves are the LLQR curves (see Appendix A). The LLQR bandwidths are 0.0942 and 0.1026 . The red lines are the linear parametric quantile regression line. The antique white curves are the asymptotic confidence band (see Appendix B) with significance level 0.05. $N=546$.

and $l(x)$ are zeros (w.r.t. $\theta)$ of the functions:

$$
\begin{aligned}
\tilde{H}_{n}(\theta, x) & \stackrel{\text { def }}{=} n^{-1} \sum_{i=1}^{n} K_{h}\left(x-X_{i}\right) \rho_{\tau}\left(Y_{i}-\theta\right) ; \\
\tilde{H}(\theta, x) & \stackrel{\text { def }}{=} \int_{\mathbb{R}} f(x, y) \rho_{\tau}(y-\theta) d y,
\end{aligned}
$$

where $\rho_{\tau}(\cdot)$ is the check function defined as (25).

THEOREM B.1. Let $h=n^{-\delta}, \frac{1}{5}<\delta<\frac{1}{3}, \lambda(K)=\int_{-A}^{A} K^{2}(u) d u$, where $K(\cdot)$ is supported on $[-A, A] . J=[0,1]$. Define $c_{1}(K)=\left\{K^{2}(A)+K^{2}(-A)\right\} / 2 \lambda(K), c_{2}(K)=\int_{-A}^{A}\left\{K^{\prime}(u)\right\}^{2} d u / 2 \lambda(K)$ and

$$
d_{n}=\left\{\begin{array}{l}
(2 \delta \log n)^{1 / 2}+(2 \delta \log n)^{-1 / 2}\left[\log \left\{c_{1}(K) / \pi^{1 / 2}\right\}+\frac{1}{2}\{\log \delta+\log \log n\}\right], \text { if } c_{1}(K)>0 \\
(2 \delta \log n)^{1 / 2}+(2 \delta \log n)^{-1 / 2} \log \left\{c_{2}(K) / 2 \pi\right\}, \text { otherwise }
\end{array}\right.
$$

Then

$$
\mathrm{P}\left[(2 \delta \log n)^{1 / 2}\left\{\sup _{x \in J} r(x)\left|l_{n}(x)-l(x)\right| / \lambda(K)^{1 / 2}-d_{n}\right\}<z\right] \rightarrow \exp \{-2 \exp (-z)\}
$$

as $n \rightarrow \infty$, with

$$
r(x)=(n h)^{1 / 2} f\{l(x) \mid x\}\left\{f_{X}(x) / \tau(1-\tau)\right\}^{1 / 2},
$$

where $f_{X}(\cdot)$ is the marginal pdf for $X$ and $f(\cdot \mid x)$ is the conditional pdf of $Y$ on $X=x$.

The corollary followed by the theorem explicitly indicates how a uniform confidence interval can be constructed. 
COROLLARY B.1. An approximate $(1-\alpha) \times 100 \%$ confidence band is

$$
l_{n} \pm(n h)^{-1 / 2}\left\{\tau(1-\tau) \lambda(K) / \hat{f}_{X}(t)\right\}^{1 / 2} \hat{f}^{-1}\{l(t) \mid t\}\left\{d_{n}+c(\alpha)(2 \delta \log n)^{-1 / 2}\right\}
$$

where $c(\alpha)=\log 2-\log |\log (1-\alpha)|$ and $\hat{f}_{X}(t), \hat{f}\{l(t) \mid t\}$ are consistent estimates for $f_{X}(t), f\{l(t) \mid t\}$.

Figure 1.1 is done by the techniques introduced in Appendices A and B. Another illustration with right tail quantiles is in Figure A.2. We plot the LLQR curve for 0.9 and 0.95 quantile. Both the two linear quantile regression lines lie outside the LLQR confidence band as the Citigroup returns are positive.

\section{PLM model estimation}

For the PLM estimation, we adopt the algorithm described in Härdle, Ritov and Song (2011). Given data $\left\{\left(X_{t}, Y_{t}\right)\right\}_{t=1}^{T}$ bivariate and $\left\{M_{t}\right\}_{t=1}^{T}$ multivariate random variables. The PLM is:

$$
y_{t}=\alpha+\beta^{\top} M_{t-1}+l\left(x_{t}\right)+\varepsilon_{t} .
$$

Let $a_{n}$ denote an increasing sequence of positive integers and set $b_{n}=a_{n}^{-1}$. For each $n=1,2, \ldots$, dividing the interval $[0,1]$ in $a_{n}$ subintervals $I_{n t}, t=1, \ldots, a_{n}$ with equal length $b_{n}$. On each $I_{n t}$, $l(\cdot)$ can approximately be taken as a constant.

The PLM estimation procedure is:

1. Inside each partition $I_{n t}$, a linear quantile regression is performed to get $\hat{\beta}_{i}$, then their weighted mean gives $\hat{\beta}$. Formally, let $\rho_{\tau}(\cdot)$ be the check function defined as $(25), l_{1}, \ldots, l_{a_{n}}$ are constants,

$$
\hat{\beta}=\underset{\beta}{\operatorname{argmin}} \min _{l_{1}, \ldots, l_{a_{n}}} \sum_{t=1}^{n} \rho_{\tau}\left\{X_{j, t}-\alpha-\beta^{\top} M_{t-1}-\sum_{m=1}^{a_{n}} l_{m} \mathbf{1}\left(X_{i, t} \in I_{n t}\right)\right\}
$$

2. Computing the LLQR nonparametric quantile estimates of $l(\cdot)$ as outlined in Appendix A from $\left\{\left(X_{i, t}, X_{j, t}-\hat{\alpha}-\hat{\beta}^{\top} M_{t-1}\right)\right\}_{t=1}^{N}$.

\section{References}

Acharya, V. V., Pedersen, L. H., Philippon, T. and Richardson, M. (2010). Measuring systemic risk, Working Paper 10-02, Federal Reserve Bank of Cleveland.

Adams, Z., Füss, R. and Gropp, R. (2010). Modeling spillover effects among financial institutions: A State-Dependent Sensitivity Value-at-Risk (SDSVaR) approach, Research Paper 10-12, European Business School.

Adrian, T. and Brunnermeier, M. K. (2011). CoVaR, Staff Reports 348, Federal Reserve Bank of New York.

Bae, K.-H., Karolyi, G. A. and Stulz, R. M. (2003). A new approach to measuring financial contagion, The Review of Financial Studies 16(3): 717-763. 
Berkowitz, J., Christoffersen, P. and Pelletier, D. (2009). Evaluating value-at-risk models with desk-level data, Working Paper 010, North Carolina State University, Department of Economics.

Brownlees, C. T. and Engle, R. (2010). Volatility, correlation and tails for systemic risk measurement, Working paper, NYU Stern School of Business.

Brunnermeier, M. and Pedersen, L. H. (2008). Market liquidity and funding liquidity, Review of Financial Studies 22: 2201-2238.

Cai, Z. and Wang, X. (2008). Nonparametric estimation of conditional VaR and expected shortfall, Journal of Econometrics 147: 120-130.

Carroll, R. and Härdle, W. (1989). Symmetrized nearest neighbor regression estimates, Statistics and Probability Letters 7: 315-318.

Chernozhukov, V. and Umantsev, L. (2001). Conditional value-at-risk: Aspects of modeling and estimation, Empirical Economics 26: 271-292.

Engle, R. and Manganelli, S. (2004). CAViaR: Conditional autoregressive value at risk by regression quantiles, Journal of Business and Economic Statistics 22: 367-381.

Fan, J., Hu, T.-C. and Truong, Y. K. (1994). Robust nonparametric function rstimation, Scandinavian Journal of Statistics 21: 433-446.

Härdle, W., Liang, H. and Gao, J. (2000). Partially Linear Models, Physica-Verlag, Heidelberg.

Härdle, W., Müller, M., Sperlich, S. and Werwatz, A. (2004). Nonparametric and Semiparametric Models, Springer-Verlag, Berlin.

Härdle, W., Ritov, Y. and Song, S. (2011). Partial linear quantile regression and bootstrap confidence bands, Journal of Multivariate Analysis . Forthcoming.

Härdle, W. and Song, S. (2010). Confidence bands in quantile regression, Econometric Theory 26: $1180-1200$.

Härdle, W., Spokoiny, V. and Wang, W. (2011). Local quantile regression, Discussion Paper 2011-005, CRC 649, Humboldt-Universität zu Berlin. Submitted to Journal of Royal Statistical Society: Series B (Statistical Method) on Feb. 1, 2012.

Hautsch, N., Schaumburg, J. and Schienle, M. (2011). Financial network systemic risk contributions, Discussion Paper 2011-072, CRC 649, Humboldt-Universität zu Berlin.

Huang, X., Zhou, H. and Zhu, H. (2011). Systemic risk contributions, Staff working papers 2011-08, The Federal Reserve Board.

Koenker, R. and Bassett, G. S. (1978). Regression quantiles, Econometrica 46: 33-50. 
Kuan, C.-M., Yeh, J.-H. and Hsu, Y.-C. (2009). Assessing value at risk with CARE, the Conditional Autoregressive Expectile models, Journal of Econometrics 150: 261-270.

Lobato, I., Nankervis, J. C. and Savin, N. (2001). Testing for Autocorrelation Using a Modified Box-Pierce Q Test, International Economic Review 42(1): 187-205.

Ruppert, D., Sheather, S. J. and Wand, M. P. (1995). An effective bandwidth selector for local least squares regression, Journal of the American Statistical Association 90: 1257-1270.

Ruppert, D. and Wand, M. P. (1995). Multivariate locally weighted least squares regression, The Annal of Statistics 23: 1346-1370.

Schaumburg, J. (2011). Predicting extreme VaR: Nonparametric quantile regression with refinements from extreme value theory, Discussion Paper 2010-009, CRC 649, Humboldt-Universität zu Berlin.

Taylor, J. W. (2008). Using exponentially weighted quantile regression to estimate value at risk and expected shortfall, Journal of Financial Econometrics 6: 382-406.

Yu, K. and Jones, M. C. (1998). Local linear quantile regression, Journal of the American Statistical Association 98: 228-237. 


\section{SFB 649 Discussion Paper Series 2012}

For a complete list of Discussion Papers published by the SFB 649, please visit http://sfb649. wiwi.hu-berlin.de.

001 "HMM in dynamic HAC models" by Wolfgang Karl Härdle, Ostap Okhrin and Weining Wang, January 2012.

002 "Dynamic Activity Analysis Model Based Win-Win Development Forecasting Under the Environmental Regulation in China" by Shiyi Chen and Wolfgang Karl Härdle, January 2012.

003 "A Donsker Theorem for Lévy Measures" by Richard Nickl and Markus Reiß, January 2012.

004 "Computational Statistics (Journal)" by Wolfgang Karl Härdle, Yuichi Mori and J ürgen Symanzik, January 2012.

005 "Implementing quotas in university admissions: An experimental analysis" by Sebastian Braun, Nadja Dwenger, Dorothea Kübler and Alexander Westkamp, J anuary 2012.

006 "Quantile Regression in Risk Calibration" by Shih-Kang Chao, Wolfgang Karl Härdle and Weining Wang, January 2012. 